\title{
A Budget Impact Analysis of the Introduction of Copanlisib for Treatment of Relapsed Follicular Lymphoma in the United States
}

\author{
Sreevalsa Appukkuttan, MPH; Emilie Duchesneau, BA; Miriam L. Zichlin, MPH; Rachel H. Bhak, MS; \\ Avin Yaldo, PhD; Mecide Gharibo, MD; Svetlana Babajanyan, MD; and Mei Sheng Duh, MPH, ScD
}

\begin{abstract}
BACKGROUND: Copanlisib was recently granted accelerated approval by the FDA for the treatment of adult patients with relapsed follicular lymphoma (FL) after 2 previous systemic therapies. It is important to assess the effect that this and other changes in the treatment landscape of relapsed FL have on a payer's budget to inform formulary decisions.

OBJECTIVE: To assess the budget impact associated with the addition of copanlisib to a formulary as third- or higher-line treatment for adult patients with relapsed FL who have received at least 2 previous systemic therapies, from the perspective of a U.S. third-party payer.
\end{abstract}

METHODS: A budget impact model was developed over a 1-year horizon. The model considered a hypothetical population of 1 million people enrolled in a commercial health plan; patients with relapsed FL were identified based on epidemiology data. Treatments included copanlisib and approved and off-label therapies used for management of relapsed FL. Treatment distributions within the target population were based on a market research survey. Drug acquisition, administration, prophylaxis, and monitoring costs were based on prescribing information, clinical trials, literature, and expert opinion. All costs were inflated to 2017 U.S. dollars. Total costs were compared between 2 scenarios, 1 without and 1 with copanlisib on a formulary. A deterministic sensitivity analysis (DSA) was conducted to evaluate the robustness of the model.

RESULTS: Within the 1 million-member health care plan, 18 patients had relapsed FL and had received at least 2 previous systemic therapies. Over 1 year, the addition of copanlisib and an increase in the use of obinutuzumab + bendamustine (from $9.0 \%$ without copanlisib to $13.1 \%$ with copanlisib) and lenalidomide + rituximab (from $0.3 \%$ to $12.0 \%$ ) were estimated to increase drug acquisition costs by $\$ 238,536$, drug administration and prophylaxis costs by $\$ 3,565$, and monitoring costs by $\$ 539$. The increase in total budget was $\$ 242,641$, corresponding to $\$ 0.02$ per member per month; $21.8 \%$ of this increase was attributable to copanlisib, $12.9 \%$ to obinutuzumab + bendamustine, and $65.3 \%$ to lenalidomide + rituximab. Results were generally robust in the DSA.

CONCLUSIONS: Over a 1-year period, the model found that the addition of copanlisib to a formulary resulted in a small increase in total budget of $\$ 242,641$, corresponding to $\$ 0.02$ per patient per month and taking into account a concurrent increase in the use of obinutuzumab + bendamustine and lenalidomide + rituximab. Therefore, adding copanlisib to a formulary appears to be an affordable option for payers. Further studies should be conducted to more comprehensively assess the clinical and economic implications of adding copanlisib to the treatment armamentarium of relapsed FL.

J Manag Care Spec Pharm. 2019;25(4):437-46

Copyright $\odot 2019$, Academy of Managed Care Pharmacy. All rights reserved.

\section{What is already known about this subject}

Treatment options for patients with follicular lymphoma (FL) who relapse after rituximab-containing regimens are limited. Copanlisib was recently granted accelerated approval by the FDA for the treatment of adult patients with relapsed FL after 2 previous systemic therapies.

The effect of changes in the treatment landscape on budget are relevant to payers when evaluating their formularies.

\section{What this study adds}

Using an economic model, this study assessed the budget impact of adding copanlisib to a formulary, as well as changes in the treatment landscape of FL, from the perspective of a U.S. third-party payer. Over a 1-year period, the model found that the addition of copanlisib to a formulary resulted in a small increase in total budget, specifically a total of $\$ 242,641$, taking into account a concurrent increase in the use of other existing treatments.

$1 \mathrm{~V}$ on-Hodgkin's lymphoma (NHL) is a group of cancers of the lymphatic system that develop from $\mathrm{B}, \mathrm{T}$, or natural killer (NK) cells. ${ }^{1}$ In the United States, NHL is the seventh most common type of cancer, accounting for more than $4 \%$ of new cancer cases and 3\% of cancer deaths in $2017 .{ }^{2}$ Depending on its clinical course, NHL is generally classified as indolent (when it grows and spreads slowly) or aggressive (when it is fast growing and symptomatic). ${ }^{3}$ The most common type of indolent NHL, and the second most common NHL overall, is follicular lymphoma (FL). ${ }^{3,4}$ Most often, FL starts from the lymph nodes and grows slowly, with 50\% of patients living more than 12 years after diagnosis. ${ }^{3}$ However, the clinical progression of FL varies from one patient to another: some have no symptoms and require no treatment for many years; others experience severe symptoms and need immediate treatment. ${ }^{5}$ When treatment is required, common options include radiation therapy (for localized disease), immunotherapy, and chemotherapy, all of which can be used alone or in combination. ${ }^{6}$ In particular, the combination of rituximab and chemotherapy has dramatically improved clinical outcomes and survival, becoming the standard of care for patients with FL in first- and second-line treatment. ${ }^{7,8}$ 


\section{FIGURE 1 Budget Impact Model Structure}

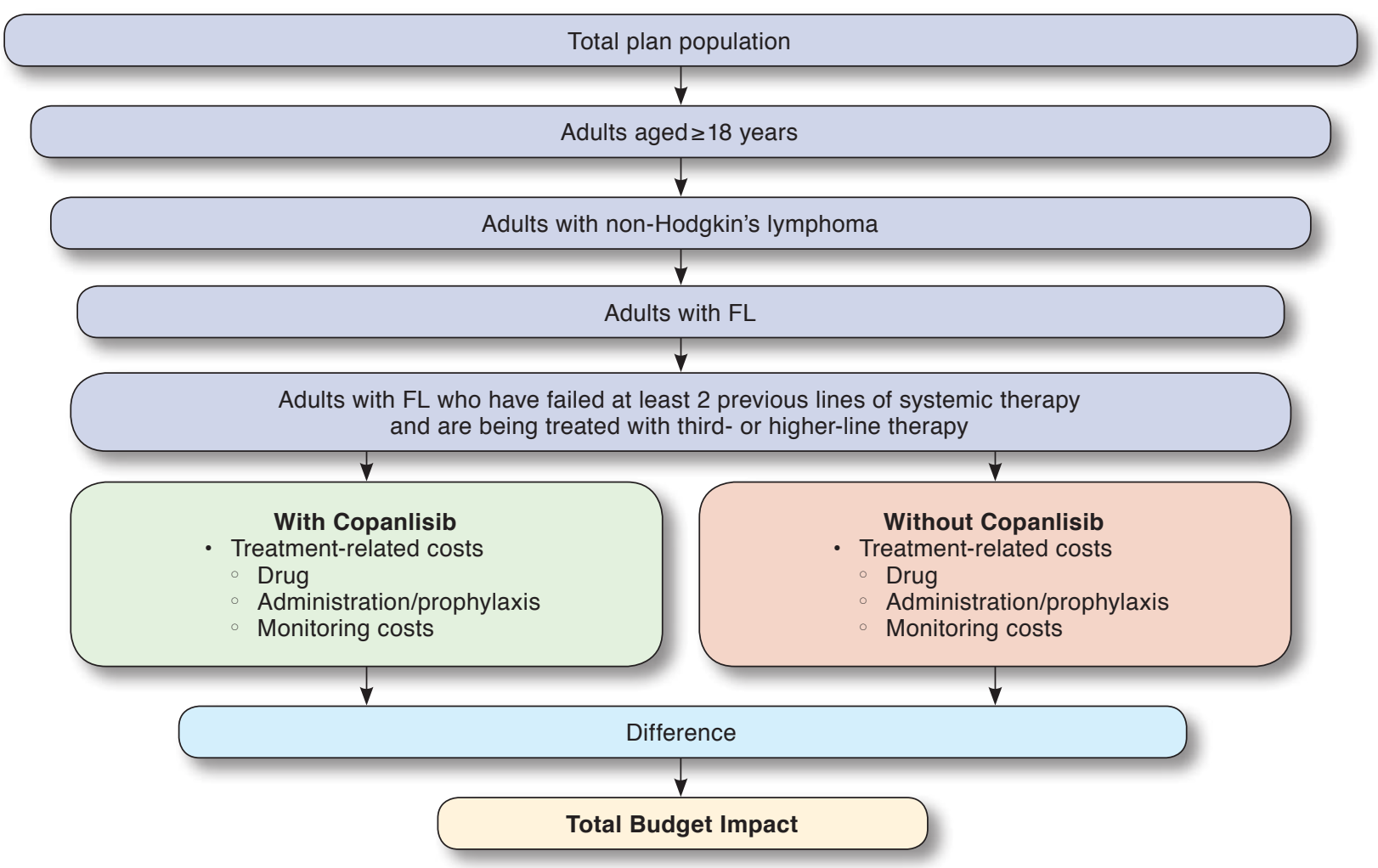

$\mathrm{FL}=$ follicular lymphoma

Despite the demonstrated clinical benefits associated with the use of rituximab in the treatment of FL, most patients experience multiple relapses, with $20 \%$ relapsing within the first 2 years of therapy based on the combination of rituximab and chemotherapy. ${ }^{9}$ For these patients, treatment options are limited. ${ }^{7}$ In 1 study, only $40 \%$ of patients with relapsed FL and previous exposure to rituximab responded to retreatment with rituximab. ${ }^{10}$ In 2013, obinutuzumab, a humanized anti-CD20 monoclonal antibody, was approved in the United States for the treatment of relapsed FL, but it has 2 black box warnings, 1 for hepatitis B virus reactivation and 1 for progressive multifocal leukoencephalopathy ${ }^{11}$ In 2014, idelalisib, a phosphatidylinositol 3-kinase (PI3K) delta isoform inhibitor, was also approved in the United States for relapsed FL in patients with at least 2 previous systemic therapies; however, recent clinical trials have shown that it is associated with an increased risk of serious adverse events such as hepatotoxicity, pneumonitis, colitis, gastrointestinal perforation, and infections. ${ }^{12}$ Another drug, the Bruton tyrosine kinase inhibitor ibrutinib, is currently listed in the most recent National Comprehensive Cancer Network (NCCN) treatment guidelines for NHL, ${ }^{6}$ but it is yet to be approved by the U.S. Food and Drug Administration (FDA) for relapsed FL, and clinical data suggest low response rates $(<17 \%)$ for patients refractory to rituximab. ${ }^{13}$

Given the elevated toxicity or low response rates of these drugs after use of rituximab, there is a need for a safer, yet effective, treatment for relapsed FL. Recently, in 2017, the FDA granted accelerated approval of copanlisib, a novel selective class I PI3K inhibitor with preferential activity against alpha and delta isoforms, for the treatment of adult patients with relapsed FL who have received at least 2 previous systemic therapies. ${ }^{14}$ Results from the open-label, uncontrolled phase 2 trial CHRONOS-1 in patients with relapsed indolent NHL (including FL) showed that copanlisib was effective as a singleagent therapy and had a manageable safety profile. ${ }^{15,16} \mathrm{Common}$ treatment-emergent adverse events included transient hyperglycemia, transient hypertension, diarrhea, fatigue, decreased neutrophil count, and fever. Copanlisib is currently listed in the NCCN guidelines for NHL as a second-line or subsequent therapy for FL refractory to at least 2 previous therapies. ${ }^{6}$

As the treatment landscape for relapsed FL continues to evolve, it is important for health care decision makers to evaluate the economic value of available treatment options and characterize the economic effect of adding new drugs to the 


\begin{tabular}{|c|c|c|}
\hline Parameter & $\mathbf{n}$ & $\%$ \\
\hline Total plan population & $1,000,000$ & 100.0 \\
\hline Adult plan members aged $\geq 18$ years 42 & 767,735 & 76.8 \\
\hline Aged $18-64$ years & 618,625 & 80.6 \\
\hline Aged $\geq 65$ years & 149,110 & 19.4 \\
\hline \multicolumn{3}{|l|}{ Prevalence of NHL among adults ${ }^{43}$} \\
\hline Aged $18-64$ years & 625 & 0.1 \\
\hline Aged 65 years & 1,429 & 1.0 \\
\hline Prevalence of FL among NHL ${ }^{44}$ & 405 & 19.7 \\
\hline $\begin{array}{l}\text { Adults with FL who have received at } \\
\text { least } 2 \text { previous therapies and initiate a } \\
\text { third or higher line of therapya, } 43,44\end{array}$ & 18 & 4.5 \\
\hline
\end{tabular}

therapeutic armamentarium of relapsed FL. Therefore, a model was developed to assess the budget impact associated with the addition of copanlisib, the latest drug approved in the United States for relapsed FL, to a formulary as third- or higher-line treatment for adult patients with relapsed FL who previously received at least 2 systemic therapies from the perspective of a U.S. third-party payer.

\section{Methods}

\section{Model Overview}

An economic model was developed in Microsoft Excel (Microsoft Corp., Redmond, WA) from a U.S. third-party payer perspective to estimate the budget impact of adding copanlisib to a formulary as third- or higher-line treatment for adult patients with relapsed FL who had at least 2 previous systemic therapies. The time horizon of the model was 1 year, which is relevant to most payers, since formularies are typically evaluated annually. Because of the short time horizon, discount rates were not considered in the analysis. The modeling approach was based on current standards for modeling the economic effect of pharmaceutical products. ${ }^{17}$

An overview of the model structure is shown in Figure 1. The model considered the size of the eligible population; the distribution of treatments; and drug, administration, prophylaxis, and monitoring costs for copanlisib and comparator treatments, which included approved treatments for relapsed FL and off-label treatments listed in NCCN guidelines (i.e., copanlisib, idelalisib, obinutuzumab + bendamustine, bendamustine + rituximab, lenalidomide + rituximab, rituximab \pm chemotherapy, and chemotherapy alone). ${ }^{6}$ In particular, the comparator rituximab \pm chemotherapy included the following regimens: rituximab alone; fludarabine + rituximab (FR); fludarabine + cyclophosphamide + rituximab (FCR); rituximab + ifosfamide + carboplatin + etoposide (R-ICE); ritux$i m a b+$ cisplatin + cytarabine + dexamethasone

(R-DHAP); rituximab + cyclophosphamide + doxorubicin + vincristine + prednisone (R-CHOP); and rituximab +cyclophosphamide+ vincristine + prednisone (R-CVP). The comparator chemotherapy alone included the following regimens: cyclophosphamide + doxorubicin + vincristine + prednisone $(\mathrm{CHOP})$ and cyclophosphamide + vincristine + prednisone (CVP).

All costs were inflated to 2017 U.S. dollars using the medical care component of the Consumer Price Index..$^{18}$

Key Model Assumptions. The model assumptions are described as follows:

- The costs associated with the treatment of adult patients with relapsed FL who received at least 2 previous systemic therapies were considered under 2 alternative scenarios: (1) without copanlisib on the formulary and (2) with copanlisib on the formulary. The budget impact was calculated as the difference in cost between the 2 scenarios. The size of the target population was assumed not to change between the 2 scenarios.

- Patients remained on copanlisib or comparators until discontinuation, which was estimated based on the median or mean treatment times reported in the literature. . $^{6,16,19-26}$ Median and mean treatment time for copanlisib were based on Bayer HealthCare Pharmaceuticals' internal Clinical Study Report PH-38670. Regimen switching was not considered. Costs of treatment, administration, prophylaxis, and services and procedures to monitor therapy-related toxicity incurred throughout the duration of treatment, which was assumed to be consistent across the 2 scenarios.

- Patients received the full dose of therapy (based on FDA prescribing information or clinical trial data [unpublished data, Bayer Healthcare Pharmaceuticals, Clinical Study Report PH-38670]) until discontinuation; dose delay, modifications, or reductions were not considered. , $, 11,12,15,16,19-24,26-41^{2}$

- Administration of intravenous infusions occurred in the inpatient setting for R-ICE and R-DHAP and in the outpatient setting for all other regimens.

- The drugs that were part of a combination therapy were administered consecutively, so the total administration time of the combination therapy was the sum of the individual administration times of all the drugs comprising the combination therapy.

\section{Model Inputs}

Target Population and Treatment Distribution. The inputs used to estimate the size of the target population are summarized in Table 1. The model considered a hypothetical population of 1 million people enrolled in a commercial health plan. Within this population, the number of adult enrollees (aged $\geq 18$ years) was estimated based on U.S. Census data. ${ }^{42}$ Among these adult enrollees, the prevalence of NHL was estimated based on the Surveillance, Epidemiology, and End Results 


\section{FIGURE 2 Treatment Distribution in Scenarios with and Without Copanlisib}

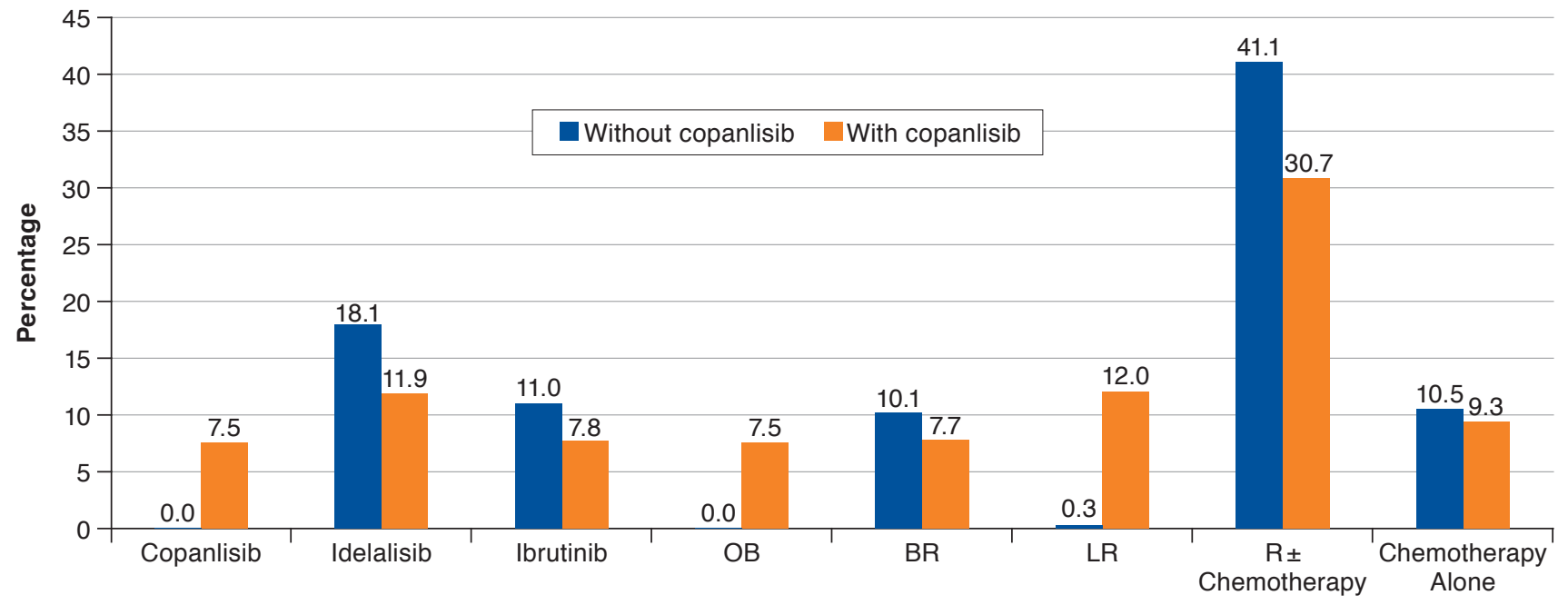

$B R=$ bendamustine + rituximab; $L R=$ lenalidomide + rituximab; $O B=$ obinutuzumab + bendamustine; $R=$ rituximab

(SEER) prevalence database. ${ }^{43}$ Among the identified adult patients with NHL, the number of those with FL was estimated based on a Datamonitor Healthcare report. ${ }^{44}$ Finally, the number of adult patients with relapsed FL (i.e., patients who initiated a third or higher line of therapy) who previously received at least 2 systemic therapies was estimated based on the SEER prevalence database, ${ }^{43}$ the Datamonitor Healthcare report, ${ }^{44}$ and internal data (unpublished data, Kantar Health, Follicular and other indolent NHL, 2017).

The proportion of patients receiving copanlisib and comparator treatments in the 2 scenarios (Figure 2) were derived from a Bayer HealthCare market research survey (fielded July 2017) of 81 oncologists in the United States treating indolent NHL and FL patients. These included 51 office-based settings and 30 hospital-based settings (20 academic/teaching hospitals and 10 community hospitals). The oncologists reported their current regimen utilization for patients treated for third-line FL (without copanlisib). Then they considered a future scenario (with copanlisib), which included all regimens selected in the scenario without copanlisib plus the blinded copanlisib option, to allow for a fair assessment of treatment options.

Costs Inputs. Cost inputs are summarized in Table 2 and described in detail below.

Drug acquisition and administration cost inputs. Drug acquisition and administration costs were estimated from the dosing schedules (i.e., drug amount and frequency) specified in prescribing

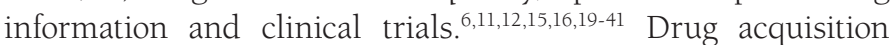
and administration costs for copanlisib were based on Bayer HealthCare Pharmaceuticals' internal Clinical Study Report PH-38670. For therapies dosed based on body surface area (BSA), the model assumed a BSA of $1.91 \mathrm{~m}^{2}$ based on mean weight and mean body mass index reported in the CHRONOS-1 trial for FL patients (unpublished data, Bayer Healthcare Pharmaceuticals, Clinical Study Report PH-38670).

Based on clinical expert input, the R-ICE and R-DHAP regimens were assumed to be administered in the inpatient setting; their treatment duration and cost were based on the literature. ${ }^{45-48}$ All the other comparator regimens were assumed to be administered in the outpatient setting.

For all the regimens, the drug cost per cycle was estimated based on the average wholesale acquisition cost reported in RED BOOK. ${ }^{49}$ Prices of generic formulations were considered, when available. Drug and drug administration costs were calculated based on mean treatment duration, which was estimated for each regimen based on information reported in clinical trials. ${ }^{6,16,19-26}$ Drug and drug administration costs for copanlisib were based on information from Bayer HealthCare Pharmaceuticals' internal Clinical Study Report PH-38670. When only the median treatment duration was reported in clinical trials, the mean treatment duration was estimated from the median duration assuming a constant rate of discontinuation.

Outpatient intravenous administration costs were obtained from the Centers for Medicare \& Medicaid Services (CMS) Physician Fee Schedule. ${ }^{50}$ Specific prophylaxis measures were based on recommendations made in the drug prescribing information. ${ }^{11,12,15,27-40}$ The unit cost for prophylactic therapy was based on the wholesale acquisition costs reported in RED BOOK and the CMS Physician Fee Schedule. ${ }^{49,50}$

Monitoring cost inputs. The cost of procedures and services to monitor therapy-related toxicity were based on recommendations made in the drug prescribing information ${ }^{11,12,15,27-40}$ and clinical 


\section{TABLE 2 Cost and Treatment Duration Inputs}

Drugs Administered in an Outpatient Setting

\begin{tabular}{|c|c|c|c|c|c|c|c|}
\hline Regimen & $\begin{array}{c}\text { Length of } \\
\text { Cycle (Days) }\end{array}$ & $\begin{array}{c}\text { Mean } \\
\text { Treatment } \\
\text { Duration } \\
\text { (Cycles) }\end{array}$ & Drug & $\begin{array}{c}\text { Drug Cost } \\
\text { per Cycle, } \$\end{array}$ & $\begin{array}{c}\text { Administration } \\
\text { Cost per } \\
\text { Cycle, } \$\end{array}$ & $\begin{array}{c}\text { Prophylaxis } \\
\text { Cost per } \\
\text { Cycle, } \$\end{array}$ & $\begin{array}{c}\text { Monitoring } \\
\text { Cost per } \\
\text { Cycle, } \$ 52,53\end{array}$ \\
\hline Copanlisiba & 28 & 7.1 & Copanlisib & 12,600 & 419 & 0 & 134 \\
\hline Idelalisib $^{12,19,49,56}$ & 30 & 9.4 & Idelalisib & 10,358 & N/A & 13 & 102 \\
\hline Ibrutinibb, 20,49 & 30 & 11.2 & Ibrutinib & 15,009 & N/A & 0 & 112 \\
\hline \multirow{2}{*}{$\mathrm{OB}^{11,21,49}$} & \multirow{2}{*}{28} & \multirow{2}{*}{6} & Obinutuzumab & 5,967 & 211 & 1 & \multirow{2}{*}{93} \\
\hline & & & Bendamustine & 8,175 & 206 & 1 & \\
\hline \multirow{2}{*}{$\mathrm{BR}^{22,29,49}$} & \multirow{2}{*}{28} & \multirow{2}{*}{5.4} & Bendamustine & 8,175 & 206 & 1 & \multirow{2}{*}{93} \\
\hline & & & Rituximab & 6,222 & 183 & 1 & \\
\hline \multirow{2}{*}{$\mathrm{LR}^{\mathrm{b}, 23,49}$} & \multirow{2}{*}{28} & \multirow{2}{*}{12} & Lenalidomide & 12,761 & N/A & 0 & \multirow{2}{*}{66} \\
\hline & & & Rituximab & 24,886 & 731 & 3 & \\
\hline \multicolumn{8}{|c|}{ Rituximab with or without chemotherapy } \\
\hline Rituximab28,49 & 7 & 4 & Rituximab & 6,222 & 183 & 1 & 104 \\
\hline \multirow{2}{*}{$\mathrm{FR}^{22,28,31,49}$} & \multirow{2}{*}{28} & \multirow{2}{*}{4.7} & Fludarabine & 517 & 345 & 0 & \multirow{2}{*}{93} \\
\hline & & & Rituximab & 6,222 & 183 & 1 & \\
\hline \multirow{3}{*}{ FCR $24,28,31,49$} & \multirow{3}{*}{21} & \multirow{3}{*}{4} & Fludarabine & 517 & 345 & 0 & \multirow{3}{*}{97} \\
\hline & & & Rituximab & 6,222 & 183 & 1 & \\
\hline & & & Cyclophosphamide & 1,003 & 198 & 90 & \\
\hline \multirow{5}{*}{ R-CHOP25,28,30,32,33,49 } & \multirow{5}{*}{21} & \multirow{5}{*}{6} & Rituximab & 6,222 & 183 & 1 & \multirow{5}{*}{112} \\
\hline & & & Cyclophosphamide & 835 & 66 & 30 & \\
\hline & & & Doxorubicin & 103 & 63 & 1 & \\
\hline & & & Vincristine & 11 & 63 & 0 & \\
\hline & & & Prednisone & 40 & N/A & 0 & \\
\hline \multirow{4}{*}{ R-CVP26,28,30,32,49,57 } & & & Rituximab & 6,222 & 183 & 1 & \\
\hline & 21 & 8 & Cyclophosphamide & 835 & 66 & 30 & 04 \\
\hline & & 8 & Vincristine & 11 & 63 & 0 & 94 \\
\hline & & & Prednisone & 31 & N/A & 0 & \\
\hline Chemotherapy & & & & & & & \\
\hline & & & Cyclophosphamide & 835 & 140 & 30 & \\
\hline CHOP25,30,32,33,49 & 21 & 6 & Doxorubicin & 103 & 63 & 1 & 112 \\
\hline $\mathrm{CHOP} 23,30,32,33,49$ & 21 & 6 & Vincristine & 11 & 63 & 0 & 112 \\
\hline & & & Prednisone & 40 & N/A & 0 & \\
\hline & & & Cyclophosphamide & 835 & 140 & 30 & \\
\hline $\mathrm{CVP}^{26,30,32,49,57}$ & 21 & 8 & Vincristine & 11 & 63 & 0 & 94 \\
\hline & & & Prednisone & 31 & N/A & 0 & \\
\hline Drugs Administered in & Inpatient Setti & & & & & & \\
\hline Regimen & $\begin{array}{l}\text { Mean } \mathrm{T}_{1} \\
\text { Duration }\end{array}$ & $\begin{array}{l}\text { atment } \\
\text { (Cycles) }\end{array}$ & $\begin{array}{r}\text { Drug } \\
\text { Prophy }\end{array}$ & $\begin{array}{l}\text { ministration, } \\
\text { s Cost per Cyc }\end{array}$ & & $\begin{array}{l}\text { Monito } \\
\text { per }\end{array}$ & $\begin{array}{l}\text { ng Cost } \\
\text { cle, } \$\end{array}$ \\
\hline R-ICE45-48 & s. & & $\begin{array}{r}20,933(5,666 \\
\text { no }\end{array}$ & $\begin{array}{l}\text { dication costs } \\
\text { dication costs }\end{array}$ & d 15,267 & & \\
\hline R-DHAP45-47 & sta & & $\begin{array}{r}16,005(5,232 \\
\text { no }\end{array}$ & $\begin{array}{l}\text { dication costs } \\
\text { dication costs) }\end{array}$ & d 10,773 & & \\
\hline
\end{tabular}

aUnpublished data, Bayer HealthCare Pharmaceuticals, Clinical study report PH-38670. Accessed April 2018.

${ }^{b}$ Not listed in NCCN guidelines for the treatment of follicular lymphoma, and its use is off label.

'Nonmedication costs include facility, labor, and laboratory costs, among others.

$B R=$ bendamustine + rituximab; $C H O P=$ cyclophosphamide + doxorubicin + vincristine + prednisone $; C V P=$ cyclophosphamide + vincristine + prednisone; $F C R=$ fludarabine + cyclophosphamide +rituximab; FR=fludarabine + rituximab; $L R=$ lenalidomide + rituximab; N/A = not applicable; NCCN = National Comprehensive Cancer Network; $O B=$ obinutuzumab + bendamustine; $R=$ rituximab; $R-C H O P=$ rituximab + cyclophosphamide + doxorubicin + vincristine + prednisone $; R-C V P=$ rituximab + cyclophosphamide + vincristine + prednisone; $R-D H A P=$ rituximab + cisplatin + cytarabine + dexamethasone; $R-I C E=$ rituximab + ifosfamide + carboplatin + etoposide. 
FIGURE 3 Base-Case Results: Total Budget Impact

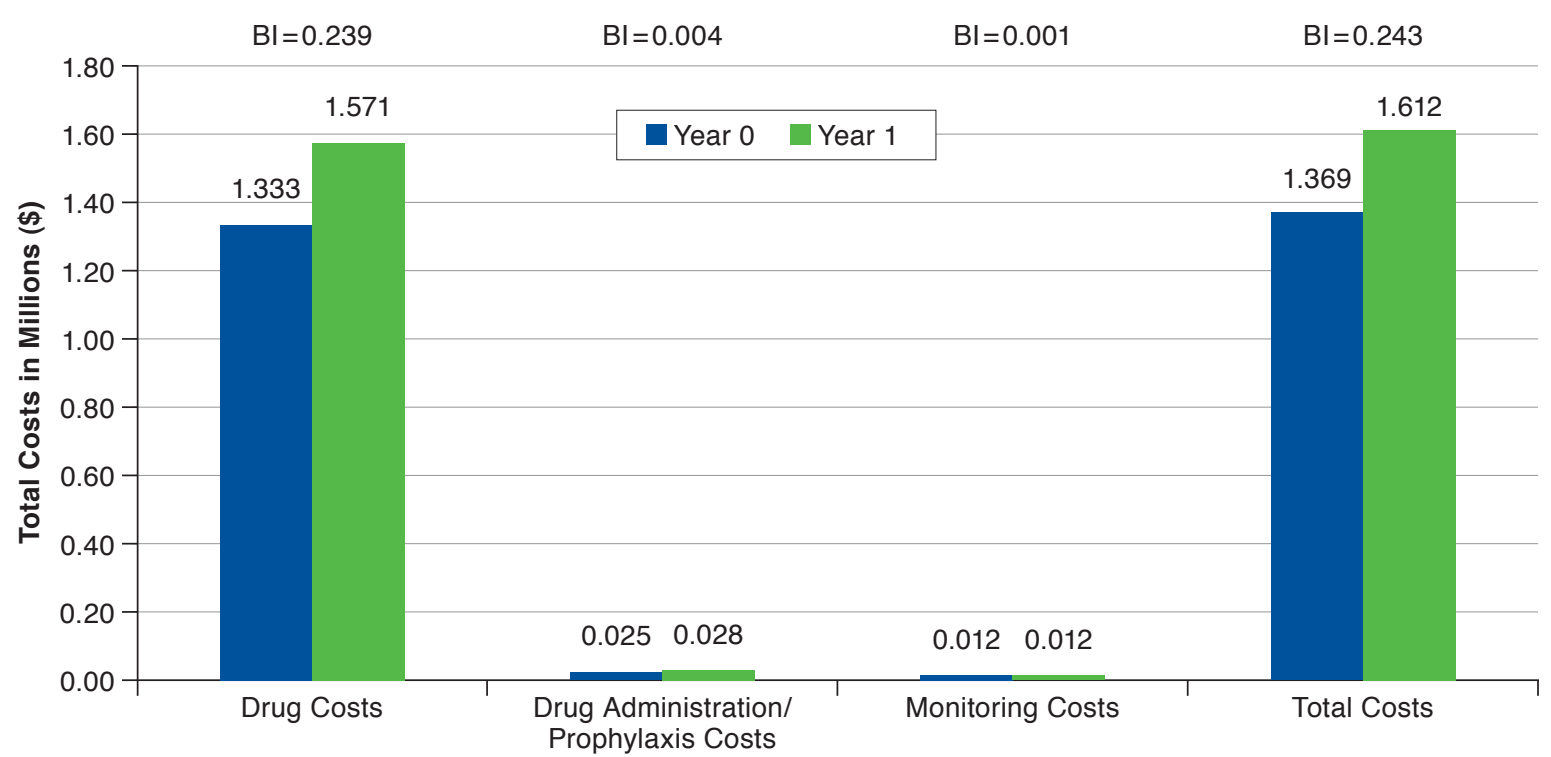

BI= budget impact.

expert opinion. The unit costs for monitoring procedures and services were obtained from CMS laboratory and physician fee schedules. ${ }^{50,51}$ Total monitoring costs per cycle were estimated for each regimen and were applied for each cycle throughout the estimated treatment duration.

\section{Model Outputs}

The model output was total costs for each scenario and budget impact, defined as the difference in costs between the 2 scenarios. To estimate these outputs, as the first step, the number of patients with relapsed FL within the 1 million-member plan was inferred from population demographics and disease prevalence, while the number of patients receiving copanlisib and the comparators in the scenarios with and without copanlisib were estimated from treatment distribution information. Subsequently, for each regimen, drug acquisition, administration, prophylaxis, and monitoring costs per cycle were multiplied by the estimated treatment duration to estimate the total treatment cost per patient over the 1-year time horizon. The total per-patient treatment cost for each regimen was then multiplied by the estimated number of patients receiving that regimen in each scenario. These total per-regimen treatment costs were summed to estimate the total costs for all the regimens for each scenario. As a last step, the total budget impact was estimated as the difference in total costs between the 2 scenarios (i.e., total costs with copanlisib minus total costs without copanlisib). The budget impact per member per month (PMPM) was calculated by dividing the total budget impact by the number of plan members and further dividing by 12 months.

\section{Deterministic Sensitivity Analysis}

A deterministic sensitivity analysis (DSA) was conducted to evaluate the robustness of the model assumptions and inputs. Specifically, model inputs were varied around their base-case values, 1 at a time, while holding other input values constant. The DSA considered assumptions related to the number of eligible patients, the cost of copanlisib and comparator regimens, treatment distribution, and duration of treatment.

\section{Results}

\section{Base Case}

In a hypothetical health plan with 1 million members, the number of adult patients with relapsed FL (i.e., who initiated a third or higher line of therapy) who received at least 2 previous systemic therapies was estimated to be 18 . In the scenario with copanlisib, it was estimated that $7.5 \%$ of these 18 patients (i.e., 1 patient) received copanlisib. In this scenario, the current offlabel regimens obinutuzumab + bendamustine and lenalidomide + rituximab were estimated to increase their U.S. market share compared with the scenario without copanlisib (from $9.0 \%$ to $13.1 \%$ and from $0.3 \%$ to $12.0 \%$, respectively).

Copanlisib, obinutuzumab + bendamustine, and lenalidomide + rituximab were estimated to have total treatment costs of $\$ 92,043, \$ 99,721$, and $\$ 178,753$, respectively, over the 1-year time horizon. The plan total cost was $\$ 1,369,421$ without 
copanlisib and \$1,612,062 with copanlisib. Because obinutuzumab + bendamustine and lenalidomide + rituximab regimens increased their market share in the scenario with copanlisib, these regimens contributed to the total costs in this scenario.

Overall, the addition of copanlisib and the increase in the market share of obinutuzumab + bendamustine and lenalidomide + rituximab were estimated to increase drug acquisition costs by $\$ 238,536$, drug administration and prophylaxis costs by $\$ 3,565$, and monitoring costs by $\$ 539$, resulting in a net increase in total budget impact of $\$ 242,641$ (Figure 3); $21.8 \%$ of the increase in total budget impact was attributable to copanlisib, $12.9 \%$ to obinutuzumab + bendamustine, and 65.3\% to lenalidomide + rituximab.

PMPM costs were estimated to equal $\$ 0.11$ without copanlisib and $\$ 0.13$ with copanlisib, with a predicted PMPM budget impact of $\$ 0.02$. Total costs by regimen can be seen in Appendix A (available in online article).

\section{Deterministic Sensitivity Analysis}

Results from the DSA were generally consistent with base-case results (Appendix B, available in online article). Total budget impact ranged from $\$ 181,980$ to $\$ 303,301$; PMPM budget impact ranged from $\$ 0.015$ to $\$ 0.025$. The model was most sensitive to the number of eligible patients and drug costs.

\section{Discussion}

Treatment options for adult patients with FL who relapse after previous systemic therapies, including rituximab-based therapies, have long been limited. ${ }^{7}$ In recent years, significant progress has been made on this front, and 3 new therapies have been approved in the United States for the treatment of relapsed FL. Therefore, it is important for payers to understand the economic implications of adding new drugs for relapsed FL to a formulary. Accordingly, this study assessed the budget impact of adding copanlisib, the latest drug approved in the United States for relapsed FL, to a formulary as third- or higher-line treatment for relapsed FL after at least 2 previous systemic therapies from the perspective of a U.S. third-party payer.

The results of this budget impact analysis showed that, in the base case, adding copanlisib to a payer formulary increased the overall budget by $\$ 242,641$ over 1 year, corresponding to an increase of \$0.02 PMPM. This increase in budget was attributable to the addition of copanlisib (which had a total treatment cost of \$92,043) and the increased use of the combination regimens obinutuzumab + bendamustine and lenalidomide + rituximab, which have total treatment costs of $\$ 99,721$ and $\$ 178,753$, respectively. These findings indicate that in a scenario where copanlisib and other regimens are available for relapsed FL, use of these regimens would increase.

Amidst changes in the treatment landscape for FL, these results are important to inform payers about the extent to which adding copanlisib to a formulary, as other available treatments increase their market share, is expected to affect their overall budget. To the best of our knowledge, this is the first study evaluating the consequences of including new and existing treatments, either approved or off-label, in third- or higherline treatment for relapsed FL in U.S. formularies. Although our results suggest that adding copanlisib to a formulary is an affordable option for payers given its low budget impact, further studies are needed to assess the cost-effectiveness of copanlisib versus other available treatments. Indeed, a budget impact analysis and a cost-effectiveness analysis are recommended by the International Society for Pharmacoeconomics and Outcomes Research to provide a comprehensive economic evaluation before formulary or reimbursement decisions. ${ }^{17,52}$ Further research is particularly important, since other therapies approved by the FDA for relapsed FL, such as obinutuzumab and idelalisib, have been associated with severe adverse events. Clinical data from CHRONOS-1 suggest that copanlisib is a safer, yet effective, treatment option for patients with relapsed FL. ${ }^{16}$ Nevertheless, more clinical trial and real-world data on copanlisib are needed to conduct additional economic analyses. Currently, two phase 3 clinical trials in relapsed FL are enrolling participants, CHRONOS-3 (NCT02367040) comparing rituximab+copanlisib with rituximab+placebo and CHRONOS-4 (NCT02626455) comparing immunochemotherapy+copanlisib and immunochemotherapy+ placebo. ${ }^{53}$ The results of these trials will provide important clinical inputs to be used in future economic models.

It is also worth noting that the FDA has recently accepted a biologics license application for a rituximab biosimilar. ${ }^{54}$ If approved, this could lead to a reduction in the cost of rituximab-based therapies, which are widely used for the treatment of FL and, in turn, to changes in formulary budgets; in this scenario, further budget impact analyses would be warranted.

\section{Limitations}

As with any economic model, the results of this budget impact model are contingent on its underlying assumptions. First, model inputs such as the size of the target population were derived from multiple data sources, and data on the prevalence of FL were limited. Nevertheless, the results of the DSA indicated that the model was generally robust to input variations, although we recognize that many of the inputs included in the model may be correlated.

Second, costs were estimated from the perspective of a U.S third-party payer, but not that of Medicare, even though the target population included patients aged $\geq 65$ years; additional economic analyses are thus needed to evaluate the budget impact from the Medicare perspective. On that same note, assessing costs (including indirect costs) from a societal perspective may also be informative to fully understand the effect of copanlisib introduction; we suggest this be revisited in future research. 
Third, the model only considered treatment, administration, prophylaxis, and monitoring costs, but there may be additional cost differences that were not accounted for, such as the cost of serious adverse events or resource utilization. This is also something that may be revisited in future research for further understanding of treatment implications. Moreover, the model also did not consider dose delays, modifications, reductions, or discounts/rebates. If the dosages received in clinical practice differ substantially from those reported in prescribing information, which was used here, the results of this study may not be generalizable to real-world practices. Similarly, if the assumption that R-ICE and R-DHAP regimens were administered in the inpatient setting while all other intravenous therapies were administered in the outpatient setting does not exactly correspond to what happens in clinical practice, the results may not be generalizable to the real world. Additionally, the internal market research study allowed oncologists to consider both on- and off-label regimens, which may have led to inflated estimates of off-label regimen use in the scenario with copanlisib. However, this would only have resulted in more conservative estimates of the budget impact, overestimating the effect of copanlisib introduction.

Finally, the applicability of the model results may change over time if there are changes in costs, treatment distribution, or target population size. Therefore, further studies should be conducted to assess any changes in budget impact over time.

\section{Conclusions}

Assuming a 1 million-member health care plan, this economic model estimated that adding copanlisib to a U.S. payer's formulary would result in a small increase in total budget $(\$ 242,641$, or $\$ 0.02$ PMPM) over a 1-year period. This increase in budget was attributable to both the addition of copanlisib and an increased use of existing combination regimens. While this study shed light on the affordability of adding copanlisib to a formulary from a payer's perspective, more studies are warranted to assess the clinical and economic implications of adding copanlisib to the treatment armamentarium of relapsed FL.

\section{Authors}

SREEVALSA APPUKKUTTAN, MPH; AVIN YALDO, PhD; MECIDE GHARIBO, MD; and SVETLANA BABAJANYAN, MD Bayer HealthCare Pharmaceuticals, Wayne, New Jersey. EMILIE DUCHESNEAU, BA; MIRIAM L. ZICHLIN, MPH; RACHEL H. BHAK, MS; and MEI SHENG DUH, MPH, ScD, Analysis Group, Boston, Massachusetts.

AUTHOR CORRESPONDENCE: Mei Sheng Duh, MPH, ScD, Analysis Group, 111 Huntington Ave., 14th Fl., Boston, MA 02199. Tel.: 617.425.8131; E-mail: Mei.Duh@analysisgroup.com.

\section{DISCLOSURES}

This study was funded by Bayer HealthCare Pharmaceuticals. The study sponsor was involved in study design, data interpretation. and data review. All authors contributed to the development of the manuscript and maintained control over the final content. Appukkuttan, Yaldo, Gharibo, and Babajanyan report employment with Bayer HealthCare Pharmaceuticals at the time of this study. Duchesneau, Zichlin, Bhak, and Duh report employment with Analysis Group, which received research funds from Bayer HealthCare Pharmaceuticals for work on this study.

A synopsis of the current research was presented in poster format at the AMCP Managed Care \& Specialty Pharmacy Annual Meeting; April 23-26, 2018; Boston, MA.

\section{ACKNOWLEDGMENTS}

Medical writing assistance was provided by Cinzia Metallo, $\mathrm{PhD}$, an employee of Analysis Group, and funded by Bayer HealthCare Pharmaceuticals. The authors also thank Adriana Valderrama for her support of this study.

\section{REFERENCES}

1. Armitage JO, Gascoyne RD, Lunning MA, Cavalli F. Non-Hodgkin lymphoma. Lancet. 2017;390(10091):298-310.

2. National Cancer Institute. Surveillance, Epidemiology, and End Results Program. Cancer stat facts: Non-Hodgkin lymphoma. 2017. Available at: https://seer.cancer.gov/statfacts/html/nhl.html. Accessed December 6, 2018

3. National Cancer Institute. Adult non-Hodgkin lymphoma treatment (PDQ)—patient version. 2018. Available at: https://www.cancer.gov/types/ lymphoma/patient/adult-nhl-treatment-pdq. Accessed December 6, 2018.

4. National Cancer Institute. What is non-Hodgkin lymphoma? 2018. Available at: https://www.cancer.org/content/cancer/en/cancer/non-hodgkinlymphoma/about/what-is-non-hodgkin-lymphoma.html. Accessed December 6, 2018.

5. Gribben JG. How I treat indolent lymphoma. Blood. 2007;109(11):4617-26 6. The National Comprehensive Cancer Network. NCCN guidelines for patients: follicular lymphoma, grade 1-2. 2017. Available at: https://www. nccn.org/patients/guidelines/nhl-follicular_lymphoma/. Accessed December 19, 2018.

7. Chao MP. Treatment challenges in the management of relapsed or refractory non-Hodgkin's lymphoma-novel and emerging therapies. Cancer Manag Res. 2013;5:251-69.

8. Dotan E, Aggarwal C, Smith MR. Impact of rituximab (rituxan) on the treatment of B-cell non-Hodgkin's lymphoma. P T. 2010;35(3):148-57.

9. Casulo C, Byrtek M, Dawson KL, et al. Early relapse of follicular lymphoma after rituximab plus cyclophosphamide, doxorubicin, vincristine, and prednisone defines patients at high risk for death: an analysis from the National LymphoCare Study. J Clin Oncol. 2015;33(23):2516-22.

10. Davis TA, Grillo-López AJ, White CA, et al. Rituximab anti-CD20 monoclonal antibody therapy in non-Hodgkin's lymphoma: safety and efficacy of re-treatment. J Clin Oncol. 2000;18(17):3135-43.

11. Gazyva (obinutuzumab) injection, for intravenous infusion. Genentech. 2013. Available at: https://www.accessdata.fda.gov/drugsatfda_docs/ label/2016/125486s013lbl.pdf. Accessed December 6, 2018.

12. Zydelig (idelalisib) tablets, for oral use. 2014. Gilead Sciences. 2014. Available at: https://www.accessdata.fda.gov/drugsatfda_docs/ label/2014/206545lbl.pdf. Accessed December 6, 2018.

13. Bartlett NL, Costello BA, LaPlant BR, et al. Single-agent ibrutinib in relapsed or refractory follicular lymphoma: a phase 2 consortium trial. Blood. 2018;131(2):182-90. 
14. Liu N, Rowley B, Bull C, Schneider C, Haegebarth A, Schatz C. BAY 80-6946 is a highly selective intravenous PI3K inhibitor with potent p110a and pl $10 \delta$ activities in tumor cell lines and xenograft models. Mol Cancer Ther. 2013;21(11):2319-30.

15. Aliqopa (copanlisib) for injection, for intravenous use. Bayer Healthcare Pharmaceuticals. 2017. Available at: https://www.accessdata.fda.gov/drugsatfda_docs/label/2017/209936s000lbl.pdf. Accessed December 6, 2018.

16. Dreyling M, Santoro A, Mollica L, et al. Phosphatidylinositol 3-kinase inhibition by copanlisib in relapsed or refractory indolent lymphoma. J Clin Oncol. 2017;35(35):3898-905.

17. Sullivan SD, Mauskopf JA, Augustovski F, et al. Budget impact analysis-principles of good practice: report of the ISPOR 2012 Budget Impact Analysis Good Practice II Task Force. Value Health. 2014;17(1):5-14.

18. U.S. Bureau of Labor Statistics. Consumer Price Index medical care component. 2017. Available at: https://data.bls.gov/timeseries/ CUUR0000SAM?output_view=data. Accessed December 19, 2018

19. Salles G, Schuster SJ, de Vos S, et al. Efficacy and safety of idelalisib in patients with relapsed, rituximab- and alkylating agent-refractory follicular lymphoma: a subgroup analysis of a phase 2 study. Haematologica. 2017;102(4):e156-59.

20. Gopal AK, Schuster SJ, Fowler N, et al. Ibrutinib as treatment for chemoimmunotherapy-resistant patients with follicular lymphoma: first results from the open-label, multicenter, phase 2 DAWN Study. Blood. 2016;128:1217

21. Sehn LH, Chua N, Mayer J, et al. Obinutuzumab plus bendamustine versus bendamustine monotherapy in patients with rituximab-refractory indolent non-Hodgkin lymphoma (GADOLIN): a randomised, controlled, open-label, multicentre, phase 3 trial. Lancet Oncol. 2016;17(8):1081-93.

22. Rummel M, Kaiser U, Balser C, et al. Bendamustine plus rituximab versus fludarabine plus rituximab for patients with relapsed indolent and mantle-cell lymphomas: a multicentre, randomised, open-label, non-inferiority phase 3 trial. Lancet Oncol. 2016;17(1):57-66

23. Leonard JP, Jung S-H, Johnson J, et al. Randomized trial of lenalidomide alone versus lenalidomide plus rituximab in patients with recurrent follicular lymphoma: CALGB 50401 (Alliance). J Clin Oncol. 2015;33(31):3635-40.

24. Sacchi S, Pozzi S, Marcheselli R, et al. Rituximab in combination with fludarabine and cyclophosphamide in the treatment of patients with recurrent follicular lymphoma. Cancer. 2007;110(1):121-28.

25. van Oers MH, Van Glabbeke M, Giurgea L, et al. Rituximab maintenance treatment of relapsed/resistant follicular non-Hodgkin's lymphoma: longterm outcome of the EORTC 20981 phase III randomized intergroup study. J Clin Oncol. 2010;28(17):2853-58.

26. Marcus R, Imrie K, Solal-Celigny P, et al. Phase III study of R-CVP compared with cyclophosphamide, vincristine, and prednisone alone in patients with previously untreated advanced follicular lymphoma. J Clin Oncol. 2008:26(28):4579-86

27. Imbruvica (ibrutinib) capsules, for oral use. Janssen Biotech. 2015. Available at: https://www.accessdata.fda.gov/drugsatfda_docs/label/2015/ 205552s002lbl.pdf. Accessed December 6,2018.

28. Rituxan (rituximab) injection for intravenous use. Genetech. 2010 Available at: https://www.accessdata.fda.gov/drugsatfda_docs/label/2010/ 103705s5311lbl.pdf. Accessed December 6, 2018.

29. Bendeka (bendamustine) injection, for intravenous use. Teva Pharmaceuticals USA. 2015. Available at: https://www.accessdata.fda.gov/ drugsatfda_docs/label/2015/208194s000lbl.pdf. Accessed December 6, 2018.

30. Cyclophosphamide injection, for intravenous use. Bayer Healthcare. 2013. Available at: https://www.accessdata.fda.gov/drugsatfda_docs/label/20 13/012141s090,012142s112lbl.pdf. Accessed December 6, 2018

31. Fludara (fludarabine) for injection; for intravenous use only. Bayer Healthcare. 2008. Available at: https://www.accessdata.fda.gov/drugsatfda_ docs/label/2009/020038s032lbl.pdf. Accessed December 6, 2018
32. VinCRIStine sulfate (vincristine) injection. Hospira. 2013. Available at: https://www.accessdata.fda.gov/drugsatfda_docs/label/2014/071484s042lbl. pdf. Accessed December 6, 2018

33. Adriamycin (DOXOrubicin $\mathrm{HCl}$ ) for injection, Bedford Laboratories 2013. Available at: https://www.accessdata.fda.gov/drugsatfda_docs/ label/2012/062921s022lbl.pdf. Accessed December 6, 2018.

34. Revlimid (lenalidomide) capsules, for oral use. Celgene. 2013 Available at: https://www.accessdata.fda.gov/drugsatfda_docs/ label/2013/021880s034lbl.pdf. Accessed December 6, 2018.

35. Ifosfamide injection. Teva Pharmaceuticals USA. 2015. Available at: https://www.accessdata.fda.gov/drugsatfda_docs/label/2015/076657s017s021 s025lbl.pdf. Accessed December 6, 2018.

36. Paraplatin (carboplatin) injection. Bristol-Myers Squibb. 2015 Available at: https://www.accessdata.fda.gov/drugsatfda_docs/ label/2010/020452s005lbl.pdf. Accessed December 6, 2018.

37. Etopophos (etoposide phosphate) for injection. Baxter Healthcare. 2010. Available at: https://www.accessdata.fda.gov/drugsatfda_docs/ label/2011/020457s013lbl.pdf. Accessed December 6, 2018.

38. Platinol (cisplatin for injection). Bristol-Myers Squibb. 2010. Available at: https://www.accessdata.fda.gov/drugsatfda_docs/label/2010/018057s079lbl. pdf. Accessed December 6, 2018

39. Cytosar-U (sterile cytarabine). Pharmacia \& Upjohn. 1997. Available at: https://www.accessdata.fda.gov/drugsatfda_docs/label/1998/016793s059lbl pdf. Accessed December 6, 2018

40. Decadron (dexamethasone tablets). Merck \& Co. 2004. Available at: https://www.accessdata.fda.gov/drugsatfda_docs/label/2004/11664slr062_ decadron_lbl.pdf. Accessed December 6, 2018.

41. Moher D, Liberati A, Tetzlaff J, Altman DG. Preferred reporting items for systematic reviews and meta-analyses: the PRISMA statement. PLoS Med. 2009;6(7):e1000097.

42. U.S. Census Bureau. Current Population Survey, Annual Social and Economic Supplement. 2016. Available at: https://www.census.gov/cps/data/ cpstablecreator.html. Accessed December 6, 2018.

43. Howlader N, Noone AM, Krapcho M, et al., eds. SEER cancer statistics review 1975-2014. Cancer prevalence tables, January 1, 2014. National Cancer Institute. Available at: https://seer.cancer.gov/archive/csr/1975_2014/ results_merged/topic_prevalence.pdf. Accessed December 19, 2018

44. Fulton Z, Dandamudi N. Epidemiology: follicular lymphoma. In: NHL Follicular Lymphoma Forecast and Market Analysis to 2025. London, UK: Datamonitor Healthcare. 2018:97-123. Available at: https://pharmastore.informa.com/product/nhl-follicular-lymphoma/. Accessed December 6, 2018.

45. Gisselbrecht C, Glass B, Mounier N, et al. Salvage regimens with autologous transplantation for relapsed large B-cell lymphoma in the rituximab era. J Clin Oncol. 2010;28(27):4184-90

46. Gisselbrecht C, Schmitz N, Mounier N, et al. Rituximab maintenance therapy after autologous stem-cell transplantation in patients with relapsed CD20(+) diffuse large B-cell lymphoma: final analysis of the collaborative trial in relapsed aggressive lymphoma. J Clin Oncol. 2012;30(36):4462-69.

47. Bodding-Long AM, Budde LE, Roden JE, Gopal AK, Chen TL. Costcomparison analysis of bendamustine, rituximab, etoposide and carboplatin (TREC) vs. RICE/R-DHAP in relapsed lymphoma. J Clin Oncol. 2015;33(15 Suppl):e19517.

48. Kewalramani T, Zelenetz AD, Nimer SD, et al. Rituximab and ICE as second-line therapy before autologous stem cell transplantation for relapsed or primary refractory diffuse large B-cell lymphoma. Blood. 2004;103(10):3684-88

49. Truven Health Analytics, IBM Watson Health. IBM Micromedex RED BOOK. Database. 2017. Available at: http://truvenhealth.com/Products/ Micromedex/Product-Suites/Clinical-Knowledge/RED-BOOK. Accessed December 7, 2018. 
50. Centers for Medicare \& Medicaid Services. Physician Fee Schedule. October 5, 2018. Available at: http://www.cms.gov/apps/physician-fee-schedule/overview.aspx. Accessed December 6, 2018.

51. Centers for Medicare \& Medicaid Services. Clinical laboratory fee schedule: 17CLAB. September 29, 2016. Available at: https://www.cms.gov/ Medicare/Medicare-Fee-for-Service-Payment/ClinicalLabFeeSched/ClinicalLaboratory-Fee-Schedule-Files.html. Accessed December 6, 2018.

52. Mauskopf JA, Sullivan SD, Annemans L, et al. Principles of good practice for budget impact analysis: report of the ISPOR Task Force on good research practices_budget impact analysis. Value Health. 2007;10(5):336-47.

53. Bayer HealthCare Pharmaceuticals. Copanlisib clinical trials. October 2018. Available at: http://www.chronostrials.com/. Accessed December 6, 2018

54. OncLive. FDA accepts application for rituximab biosimilar. September 12, 2017. Available at: https://www.onclive.com/web-exclusives/fda-acceptsapplication-for-rituximab-biosimilar. Accessed December 6, 2018.
55. Bactrim (sulfamethoxazole and trimethoprim) injection, for intravenous use. Sun Pharmaceutical Industries. 2017. Available at: https://www. accessdata.fda.gov/drugsatfda_docs/label/2017/018374s025lbl.pdf. Accessed December 6, 2018.

56. Gilead. Direct healthcare professional communication: restrictions on the use of Zydelig (idelalisib) for the treatment of chronic lymphocytic leukaemia (CLL) and relapsed follicular lymphoma (FL) following new clinical trial results. Gilead submission reference: 1101-16-208. March 23, 2016. Available at: https://assets.publishing.service.gov.uk/ media/5707baba40f0b60385000056/Zydelig__idelalisib____DHPC_ sent_23_03_2016.pdf. Accessed December 6, 2018.

57. Marcus R, Imrie K, Belch A, et al. CVP chemotherapy plus rituximab compared with CVP as first-line treatment for advanced follicular lymphoma. Blood. 2005;105(4):1417-23. 
APPENDIX A Base-Case Results: Total Costs by Regimen in Scenarios with and Without Copanlisib

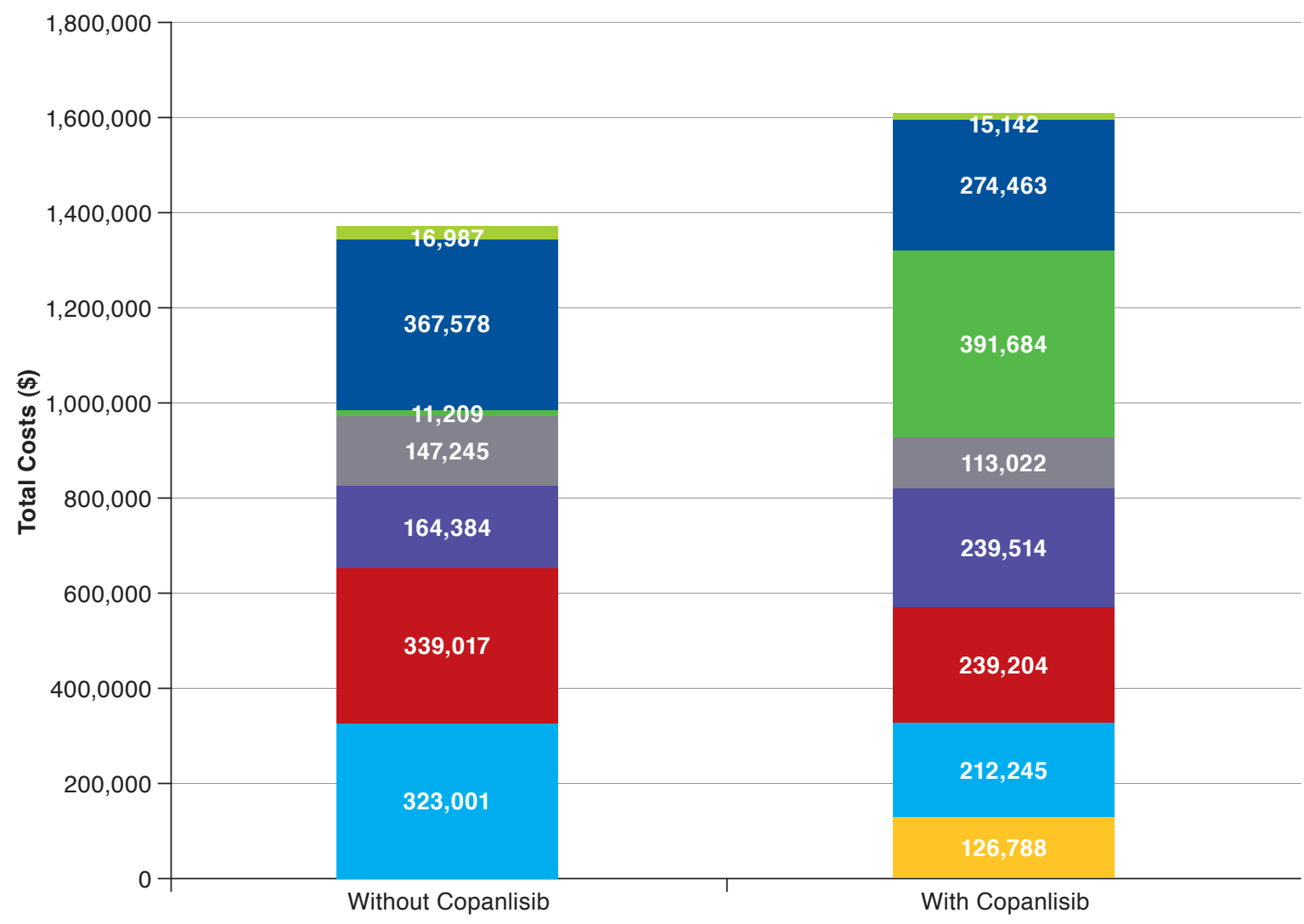

Copanlisib Idelalisib $\square$ Ibrutinib $\quad$ OB $\square$ BR $\quad$ LR $\quad \mathrm{R} \pm$ chemotherapy $\square$ Chemotherapy alone 
A Budget Impact Analysis of the Introduction of Copanlisib for Treatment of Relapsed Follicular Lymphoma in the United States

APPENDIX B Deterministic Sensitivity Analysis Results

Total Budget Impact (\$ millions)

$\begin{array}{llllllll}0.000 & 0.050 & 0.100 & 0.150 & 0.200 & 0.250 & 0.300 & 0.350\end{array}$

Total number of eligible patients $( \pm 25 \%)$

Copanlisib drug costs $( \pm 25 \%)$

Drug costs of other comparators $( \pm 25 \%)$

Duration of treatment based on median

Body surface area $( \pm 25 \%)$

Total number of patients taking copanlisib in Year $1( \pm 25 \%)$

Use brand WAC price

Copanlisib monitoring cost $( \pm 25 \%)$

Monitoring cost of other comparators $( \pm 25 \%)$

Decrease in input value

Increase in input value

WAC $=$ wholesale acquisition cost. 